\title{
ERG induces a mesenchymal-like state associated with chemoresistance in leukemia cells
}

\author{
Liliana H. Mochmann ${ }^{1}$, Martin Neumann ${ }^{1}$, Eva K. von der Heide ${ }^{1}$, Verena Nowak ${ }^{2}$, \\ Anja A. KühI ${ }^{3}$, Jutta Ortiz-Tanchez ${ }^{1}$, Juliane Bock ${ }^{1}$, Wolf K. Hofmann' ${ }^{2}$, Claudia D. \\ Baldus ${ }^{1}$ \\ ${ }^{1}$ Department of Hematology and Oncology, Charité University Medicine Berlin, Berlin, Germany \\ 2 Department of Hematology and Oncology, University Hospital Mannheim, Mannheim, Germany \\ ${ }^{3}$ Department of Gastroenterology, Infectiology and Rheumatology, Charité University Medicine Berlin, Berlin, Germany \\ Correspondence to: Claudia D. Baldus, email: claudia.baldus@charite.de
}

Keywords: ERG, ERK, EMT, Chemoresistance

Received: September 26, 2013 Accepted: December 3, $2013 \quad$ Published: December 4, 2013

This is an open-access article distributed under the terms of the Creative Commons Attribution License, which permits unrestricted use, distribution, and reproduction in any medium, provided the original author and source are credited.

\section{ABSTRACT:}

Overexpression of the oncogene ERG (ETS-related gene) is an adverse prognostic factor in acute myeloid and T-cell lymphoblastic leukemia (AML and T-ALL). We hypothesize that ERG overexpression is associated with primary drug resistance thereby influencing the outcome in leukemia. We previously reported a cell-line based model of ERG overexpression which induced a potentially chemo-resistant spindle shape cell type. Herein, we report a specific transcriptional gene signature for the observed spindle shaped morphology. Genes significantly over-expressed after ERG induction strongly resembled adhesive mesenchymal-like genes that included integrins (ITGA10, ITGB5, ITGB3, ITGA2B), CD44, and CD24. Interestingly, the mesenchymallike signature was accompanied by the repression of DNA chromatin remodeling and DNA repair genes, such as CHEK1, EZH2, SUZ12, and DNMT3a. The ERG-induced mesenchymal-like signature positively correlated with TMPRSS2-ERG prostate tissues and invasive breast cancer mRNA expression datasets reflecting a general ERG-driven pattern of malignancy. Furthermore, inhibitors modulating ERG druggable pathways WNT, PKC, and AKT, and chemotherapeutic agent cytarabine revealed ERG-induced drug resistance. In particular, PKC412 treatment enhanced proliferative rates and promoted spindle shape formation in ERG-induced cells. Nilotinib and dasatinib were effective at abolishing ERG-induced cells. Moreover, ERG overexpression also led to an increase in double strand breaks. This report provides mechanistic clues into ERG-driven drug resistance in the poor prognostic group of high ERG expressers, provides insight to improved drug targeted therapies, and provides novel markers for a mesenchymal-like state in acute leukemia.

\section{INTRODUCTION}

The oncogene ERG belongs to an evolutionary related group of ETS DNA binding proteins and directs gene expression in hematopoietic processes establishing definitive hematopoiesis, maintaining the stem cell pool[1] and promoting megakaryocytic differentiation[2]. Chromosomal aberrations harboring a fusion product of ERG to form FUS/TLS-ERG in acute myeloid leukemia (AML)[3], ERG-EWS in Ewing's sarcoma[4], or
TMPRSS2-ERG[5,6] in prostate cancers are predictive of poor prognosis. Likewise, high levels of ERG correlate with a worse outcome in cytogenetically normal AML and acute T-lymphoblastic leukemia (T-ALL)[7,8]. Mouse models overexpressing Erg clearly revealed an oncogenic phenotype, with high Erg causing fetal hematopoietic progenitors to develop leukemia[2]. Similarly, high ERG expressing bone marrow cells transplanted in adult mice produced Notch1 mutations and T cell expansion[9]. Recently it was reported that about $30 \%$ of transgenic 
ERG mouse models develop T-ALL[10] whereas the remainder develop myeloid leukemia at five months[11].

Current chemotherapy regimens are insufficient for high-risk acute leukemia patients characterized by high ERG expression. For instance, in AML, the cumulative incidence of relapse in high ERG expressers was $81 \%$, in comparison to only $33 \%$ in low ERG expressers at 5-years[7]. Similarly, the overall survival of high ERG expressers in T-ALL at 5 year years is only $26 \%$ versus $58 \%$ in low ERG expressers[8]. Thus, understanding the ERG gene regulatory networks responsible for treatment failure and involved in drug resistance at the molecular level will aid in understanding the etiology of high ERG expression in acute leukemia.

Due to the high incidence of TMPRSS2-ERG fusion in prostate cancer, recent studies have mainly focused on mapping ERG signaling networks in prostate. These networks comprise a diaspora of functions that show a role for ERG in the regulation of extracellular matrix through the plasminogen activator pathway[12], upregulation of epithelial-to-mesenchymal transition (EMT) genes[13], ERG-mediated regulation of chromatin though binding to the EZH2 promoter, and DNA repair regulation through poly (ADP-ribose) polymerase (PARP) interactions[14]. This composite ERG gene signatures correlates well with the clinical characteristics of prostate cancer, and is thought to contribute to disease progression in prostate cancer[15,16].

While it is unarguable that ERG overexpression is involved in oncogenesis of leukemia and prostate cancers, much less is clear as to how ERG signaling mediates drug resistance. Emerging reports describe EMT in tumor progression as a mechanism for cell proliferative and survival advantages[17]. EMT is defined as an epithelial cell undergoing transformation, acquiring mesenchymal-like features that allow a cell to be motile and able to migrate. This process requires specific changes in gene regulation and is remarkably reversible (termed mesenchyme-to-epithelial, MET) via epigenetic changes[18]. Moreover, the acquisition of mesenchymelike (derived from MET) properties in both malignant cells and non-epithelial cells has been proposed as a mechanism for drug resistance in solid tumors of the lung, breast, prostate[18] and in chronic myeloid leukemia[17]. Several ETS transcription factors have been implicated in turning on an EMT-like program and, likewise, studies in cancer cells document enhanced cell migration in EMT overexpressing cells[19,20]. Taken together, these studies support the notion that EMT in high ERG expressers may contribute to drug resistance in prostate carcinoma. Herein, we report that in leukemia, ERG overexpression causes molecular characteristics that are strikingly similar to the ERG-associated signaling networks in prostate cancer. ERG overexpression induces a mesenchymal-like state with a highly drug resistant phenotype, pronounced proliferative growth advantage, and promotes double strand breaks (DSBs) formation. Our findings may have important clinical implications for the improvement of current therapies in adult leukemia.

\section{RESULTS}

\section{ERG induction promotes mesenchymal-like gene expression signature accompanied by repression of DNA remodeling and DNA repair}

We previously reported that prolonged ERG overexpression induced leukemia cells to adhere and develop bi-directional protrusions (spindle shaped cells) (Fig. 1A). This morphogenic state was in part attributable to the upreguation of WNT11[21]. However the overall transcriptional program for this morphogenesis had not been characterized (Fig 1A). Herein, we sought to elucidate the morphogenesis program responsible for the spindle shape formation upon ERG induction by determining the global transcription profile of leukemic K562 cells harbouring tet-on inducible ERG expression constructs. When comparing global gene expression of ERG-induced cells versus non-induced cells, 128 genes were differentially over-expressed whereas 1440 were differentially under-expressed (Figure 1B, fold change $\geq 2$; $<<0.05$ ). Through Gene Ontology (GO) analyses, the over-expressed genes were significantly enriched for cell adhesion, migration, and motility (Table 1, top), a pattern that highly resembled the adhesive EMT properties and included integrin upregulation. We verified by PCR upregulation of gene products associated with biological adhesion and morphogenesis including: CD24, CD44, ITGA10, PLAUR, CXCL11, SELP, TYROBP, FLT4, and SHANK3 (Figure 1C). While the EMT/MET genetic program has yet to be fully understood in cancer, recent studies showed that upregulation of two novel EMT key markers, CD44 and CD24, are necessary for entry into a mesenchymal-like state in highly drug resistant breast HER2 positive carcinoma[25]. Contrary to typical EMT/ MET, the EMT markers E- and N-cadherin were not differentially altered by ERG overexpression, suggesting that the EMT/MET gene signature may be different in leukemia than in breast cancer. NUMB, both a binding target of ERG[26] and reported to be involved in EMT regulation[27], was also under-expressed (Figure 1C). Additional ERG under-expressed genes were enriched for the GO term "DNA homologous recombination and repair" including the CHEK1, ATM, and BRCA1 DNA repair genes (Table 1, bottom). DNA chromatin remodeling proteins, such as EZH2, SUZ12, and DNMT3a, were also under-expressed (Figure 1C), as observed in prostate cancer studies[14,28]. Hence, repression of DNA repair 
and DNA chromatin remodeling genes may provide a link to the molecular mechanisms through which ERG overexpressing cells induce a mesenchymal-like state.

We then used Oncomine, a microarray cancer database, to assess whether ERG-induced gene signature generated from the K562 pTRE-ERG leukemia cell line A
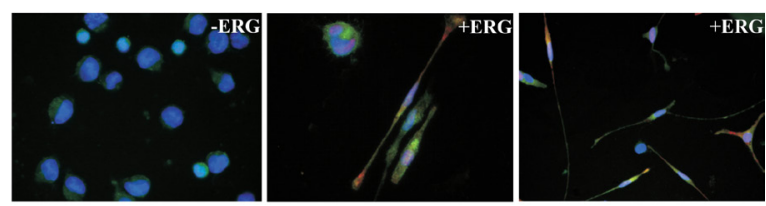

B.

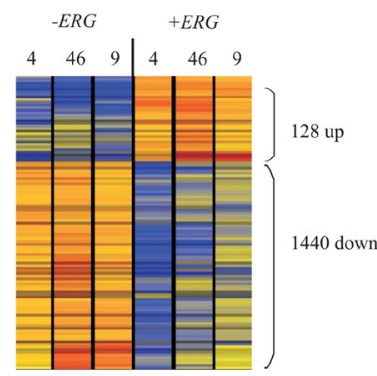

C.

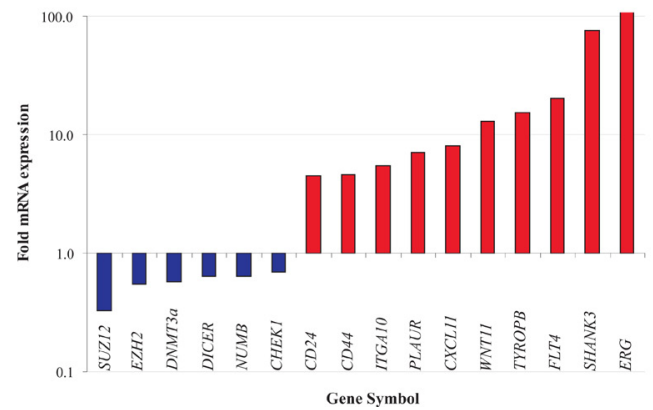

Figure 1: ERG overexpression induces a mesenchymallike signature accompanied by repression of DNA homologous recombination repair gene expression. A) Fluorescence imaging of ERG-induced cells (+ ERG, DsRed fluorescence) developing spindle shape formation following DOX stimulation for 96 hours. Rabbit anti-WNT11 antibody (1:100) coupled with goat anti-rabbit IgG-FITC (1:100) was used for colocalization with DsRed positive (+ERG). DAPI $(1: 100)$ was used to stain the nuclei. Non-induced (-ERG, left) maintain the native round morphology with basal levels of WNT11 on the cell surface. WNT11, a direct target of ERG, localizes to the nuclei and along the spindle branches (center, 400X magnifications and right, 200X magnification). B) Heat map displaying the ERG gene signature derived from three stable transfected K562 pTRE-ERG clones (CL4, CL46 and CL9, referred to as 4, 46 and 9) induced with DOX for 6 days. Red to orange hues, strong expression; blue to yellow hues, low expression of induced cells relative to non-induced cells. A 2-fold cut off was used to determine the significance of overexpressed (128) and under-expressed genes (1440). C) RT PCR validation of novel ERG transcriptional targets from Figure 1B related to kinase, DNA modification, or adhesion. Fold mRNA expression (y-axis) of each gene reports the ratio between induced and non-induced ERG mRNA expression. RT PCR was conducted in duplicate and bar graphs represent the data for the CL9 K562 pTRE-ERG clone. was disease-relevant. 128 over-expressed genes were uploaded to Oncomine platform. ERG-induced gene signature positively correlated with three invasive and metastatic cancer mRNA expression datasets: 1) Grasso dataset prostate tissues habouring ERG rearrangement versus no ERG rearrangement[29], 2) Grasso dataset of prostate carcinoma tissues versus normal prostate gland[29] and 3) Stickeler dataset of invasive breast cancer patients treated with a standard regimen of chemotherapy assessing post-chemotherapy versus pretreatment changes[30] (Supplement Figure 1A-D and Supplement Table 1). Of note, in the latter dataset, 17 of 25 patients with invasive breast cancer did not result in complete remission indicating that the analysis of transcriptionally deregulated genes could aid in predicting the clinical response to cancer treatment. Recent studies in a prostate cell line over-expressing ERG also concur with our results, i.e. they exhibit highly enriched cell adhesion and cell migration GO terms[31] and promote EMT gene expression[32], thus providing further support for an ERG-driven mesenchymal-like state in leukemia. Taken together, the positive correlation of ERG-induced mesenchymal-like gene signature in leukemia with solid tumor prostate cancer tissues demonstrate that an ERGspecific gene signature is induced, which is independent of disease origin, and may provide sensitive molecular targets of prognostic value, thereby aiding in predicting the outcome or response to therapy.

\section{ERG overexpression results in a profound apoptotic resistance}

Recently, we reported that ERG overexpression conferred resistance to the multi-kinase inhibitors TKI258 and sorafinib[26]. A panel of 15 drugs was selected to further explore the ERG-driven drug resistance. From our own studies in leukemia cells and previous reports in prostate cancer genes, we selected the ERG-targeted networks to include: WNT signaling[21], AKT/PI3K signalling[26,33], ERK[34], DNA repair[35], and DNA chromatin remodeling (Table 2)[36]. ERG-induced drug resistance was determined by three criteria: 1) the significant difference in apoptotic induction in noninduced cells in comparison with ERG-induced cells, 2) the maintenance or expansion of ERG-induced cells (DsRed positive and absence of apoptosis), 3) by the persistence of spindle shape cells upon drug treatment (Table 2). Under those conditions, ERG-induced cells were considered resistant to the following agents: LY294002, rapamycin, quercetin, PKC412, cytarabine (Ara-C), ICG001, and TKI258 as previously determined[26] (Figure 2A, upper panel). Remarkably, ERG-induced cells were not only resilient to apoptosis when treated with Ara-C, LY294002, or PKC412 but also displayed sustained DsRed population upon Ara-C treatment and expansion 
Table 1. ERG overexpression results in a mesenchymal-like signature accompanied by dowreguation of DNA repair. Significantly over-expressed and underexpressed genes were uploaded and analyzed with DAVID Bioinformatics. Listed below are genes that signficantly cluster to the respective gene ontology functions. P-Value is included for significance.

\begin{tabular}{lcll}
\multicolumn{1}{c}{ GOTERM (Over-expressed) } & Count & P-Value & Gene Symbol \\
\hline integrin-mediated signaling pathway & 4 & $2.58 \mathrm{E}-03$ & ITGA10, ITGB5, ITGB3, ITGA2B, FLT4 \\
chemotaxis & 5 & $3.52 \mathrm{E}-03$ & CXCL1, SEMA3F, CXCL11, PLAUR, CMTM5 \\
response to wounding & 8 & $4.40 \mathrm{E}-03$ & CXCL1, AIF1, CLU, GP1BA, ITGB3, CXCL11, TIMP3, PLAUR \\
cell-matrix adhesion & 4 & $5.09 \mathrm{E}-03$ & ITGA10, ITGB5, ITGB3, ITGA2B \\
cell-substrate adhesion & 4 & $6.65 \mathrm{E}-03$ & ITGA10, ITGB5, ITGB3, ITGA2B \\
immune response & 8 & $1.74 \mathrm{E}-02$ & CXCL1, LST1, CD300A, CLU, FCERIG, MYO1F, CXCL11, ARHGDIB \\
cell adhesion & 8 & $1.87 \mathrm{E}-02$ & CD300A, PECAM1, GPR56, ITGA10, ITGB5, GP1BA, ITGB3, ITGA2B \\
locomotory behavior & 5 & $2.22 \mathrm{E}-02$ & CXCL1, SEMA3F, CXCL11, PLAUR, CMTM5 \\
response to organic nitrogen & 3 & $2.53 \mathrm{E}-02$ & ALDOC, PTGS1, TIMP3 \\
regeneration & 3 & $2.99 \mathrm{E}-02$ & ALDOC, TIMP3, PLAUR \\
wound healing & 4 & $3.88 \mathrm{E}-02$ & GP1BA, ITGB3, TIMP3, PLAUR \\
leukocyte mediated immunity & 3 & $4.47 \mathrm{E}-02$ & $C L U, F C E R 1 G, M Y O 1 F$ \\
\hline \multicolumn{1}{c}{ BIOCARTA (Under-expressed) } & Count & P-Value & \\
\hline Role of BRCA1, BRCA2 and ATR in Cancer & 5 & $3.23 \mathrm{E}-02$ & LOC651610, LOC648152, LOC651921, CHEK1, ATR, ATM, RAD50, BRCA1 \\
\hline
\end{tabular}

of the DsRed population upon PKC412 or LY294002 treatment (Figure 2A, lower panel). Moreover, treatment with AKT inhibitor, LY294002, or PKC inhibitor, PKC412 enhanced spindle shape formation (Table 2). Conversely, ERG-induced cells were sensitive to daunorubicin, dasatinib, nilotinib, and 5-azacytidine as assessed from the diminishing DsRed positive population, and increasing
Annexin V-FITC population. The extent of cell death upon PD325901 treatment between ERG-induced and non-induced cells did not differ; however given that the DsRed positive population was depleted, ERG-induced cells were considered sensitive to PD325901. Drugs that did affect apoptosis in both ERG-induced and non-induced cells were 3-deazaneplanocin A and olaparib (AZD2281).
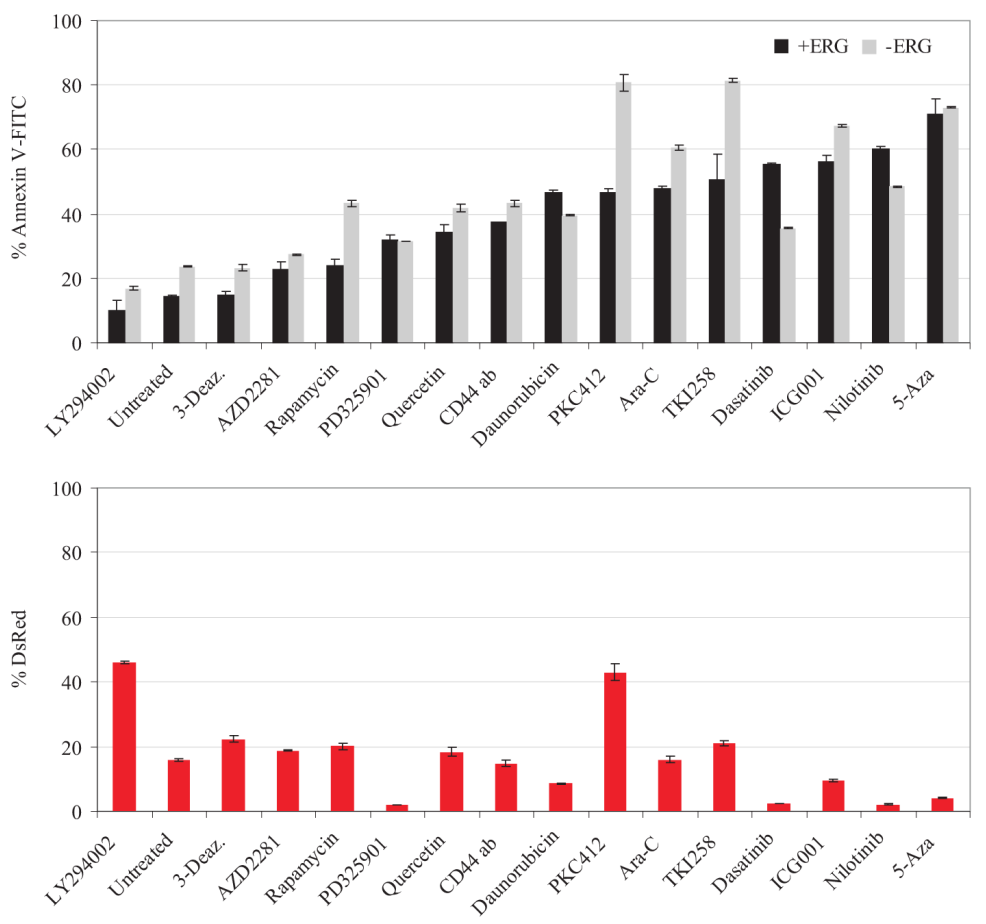

Figure 2: Drugs testing various branches of ERG targeted pathways elicit ERG-mediated drug resistance, which is enhanced by the presence of HS-5 and AML MSCs. A panel of 15 drugs (Table 2) was surveyed to determine the extent of ERG dependent drug resistance. ERG-induced and non-induced cells were treated with 15 drug candidates aimed at inducing apoptosis and traced by Annexin V-FITC. Values plotted are the total Annexin V-FITC positive cell population of ERG-induced (+ERG, black bars) and non-induced cells (-ERG, grey bars). Upper plot, ranking by ERG resistance to apoptosis; lower plot, ERG-induced cells (non-apoptotic) were tracked by DsRed fluorescence (+ERG, red bars). Representative results for the CL9 clone are plotted. The error bars denote the standard deviation 
Table 2. ERG transcriptional pathways are targeted with specific compounds to determine the resiliency of ERG dependent spindle shape formation.

\begin{tabular}{|c|c|c|c|}
\hline Compound & Category & Mode of Action & Spindle Shape Formation \\
\hline Cytarabine (Ara-C) & DNA modulation & $\begin{array}{l}\text { incorporation into DNA, inhibition of DNA } \\
\text { polymerase }\end{array}$ & ++ \\
\hline Daunorubicin & & interacts with DNA by interchalation & - \\
\hline Olaparib (AZD2281) & & inhibiotor of PARP & - \\
\hline 3-deazaneplauocin & & inhibitor of EZH2 & - \\
\hline 5-Aza-deoxcytidine (5-Aza) & & inhibitor of DNA methylation & ++ \\
\hline TKI258* & kinase inhibitors & $\begin{array}{l}\text { inhibitor of FLT3, c-KIT, FGFR, VEGFR } 1 / 2 / 3 \text {, } \\
\text { PDGFRß and CSF-1R }\end{array}$ & + \\
\hline Nilotinib & & inhibitor of BCR-ABL tyrosine kinase & - \\
\hline Dasatinib & & inhibitor of BCR-ABL and SRC tyrosine kinase & - \\
\hline PKC412 & & inhibitor of FLT3, PKC, PDGFR, VEGFR2 & ++ \\
\hline PD325901 & ERK inhibitor & inhibitor of ERK & + \\
\hline Rapamycin & AKT modulation & $\begin{array}{l}\text { complexes with FKBP12, indirect inhibitor of } \\
\text { mTOR }\end{array}$ & ++ \\
\hline LY294002 & & inhibitor of PI-3 kinase & ++ \\
\hline 6-bromoindirubin-3'-oxime* & WNT modulation & inhibitor of GSK-3b, activation of WNT pathway & - \\
\hline Quercetin & & inhibition of WNT pathway & + \\
\hline XAV939 & & inhibition of WNT pathway & ++ \\
\hline ICG001 & & inhibition of WNT pathway & ++ \\
\hline CD44 & blocking antibody & blocks cell to cell interactions & - \\
\hline
\end{tabular}

The lack of 3-deazaneplanocin A (EZH2 inhibitor) [36] and AZD2281 (PARP inhibitor)[37] effectiveness in ERG-induced cells may be due to repression of the drug targets EZH2 and CHEK1. We conclude that ERGinduced cells are resistant to Ara-C, WNT inhibitors, drugs targeting the protein kinase $\mathrm{C}$ pathway (PKC412) and downstream effectors: i.e. the PI3K inhibitors LY294002 and rapamycin. Thus, PKC kinase inhibitors, WNT inhibitors and Ara-C are predicted not to be effective in clearing ERG overexpressing cells in leukemia. By contrast, nilotinib is expected to reverse drug resistance, by selectively enhancing apoptosis most potently in cell model system overexpressing ERG.

\section{ERG overexpressing cells cultured on primary AML-derived stroma have a proliferative growth advantage}

Given the survival properties of ERG overexpression, we examined the proliferative properties of ERG-induced cells. CFSE labeled ERG-induced cells displayed a 2-fold proliferative advantage over non-induced cells at 72-hours (Figure 2B). Proliferative growth was further enhanced by PKC412 treatment in ERG-induced cells over non-induced cells, and treatment with Ara-C resulted in minimal advantage over the noninduced group. To further monitor this proliferative advantage, co-cultured ERG-induced cells were harvested in direct contact with the HS-5 stromal layer. Also in this case, ERG-induced cells manifested a 2-fold greater rate of proliferation than cells cultured without stroma (Figure 2B). We further tested the proliferative effect of the direct contact of stroma mesenchymal cells with ERG-induced cells from three AML donors, which confirmed a similar rapid decay rate of CFSE, as observed with the HS-5 coculture. These results demonstrate a synergistic interaction between ERG overexpressing cells and the contacting HS-5 stroma, or primary AML MSC.

We also investigated ERG-overexpressing cells treated with PKC412, to test the effect of drug resistance in the presence of HS-5 and AML MSCs. Untreated ERG DsRed positive cells were expanded in the presence of HS-5 (10\%) but not in the presence of AML MSCs. However, ERG-induced cells (DsRed positive) treated with PKC412 expanded by $40 \%$ in co-culture with HS-5 and 20\% with AML MSCs (Figure 2C). Thus, ERGdependent resistance to apoptotic induction was enhanced via stroma protection through cell-to-cell contacts upon PKC412 treatment, in comparison with $80 \%$ apoptotic induction in non-induced cells.

\section{Blocking CD44 by antibody binding inhibits ERG-induced spindle shape morphology}

Due to the enhanced survival of ERG-induced cells, we sought to identify potential adhesion molecules induced by ERG overexpression that would regulate cell-to-cell contacts. A key regulator of mesenchymallike properties is CD44, a major adhesion molecule for homing, cell migration, and signal transduction[18]. Deregulated CD44 contributes to leukemogenesis, and blocking CD44-mediated adhesion by complexing antibodies to aid in the detachment of leukemia cells from the bone marrow niche has been proposed as a targeted 
therapy in leukemia[38]. CD44 was significantly overexpressed (2-fold) in the ERG-induced clones. Therefore, we tested a CD44 blocking antibody in culture with

A.

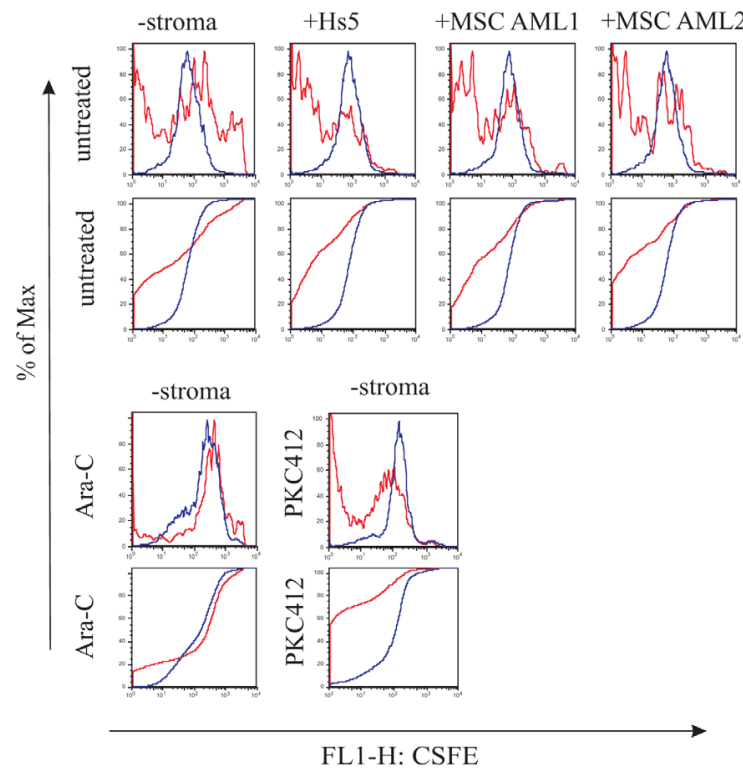

B.

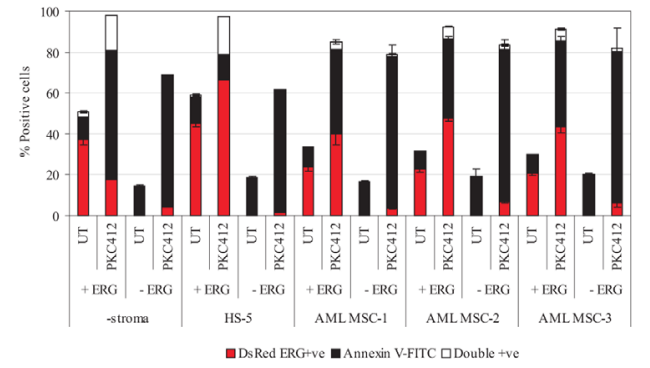

Figure 3: ERG-mediated drug resistance is enhanced by the presence of HS-5 and AML MSCs. A) Histograms displaying the decay of CFSE dye as a function of cell division (proliferation) of ERG-induced (+ERG, DsRed positive and CFSE) and non-induced (-ERG, DsRed negative and CFSE) cells. Co-culture in the presence of HS- 5 stroma or primary AMLderived MSCs was for 96 hours. Plotted below each histogram is the cumulative distribution function (CDF) plot (black) or the fluorescence distribution (red) for visualization of CFSE decay rates of +ERG versus -ERG cells in the presence of HS-5, AML MSCs, or treatment with Ara-C or PKC412. B) ERG-inducible (+ERG) and non-inducible (-ERG) cells were treated with PKC412 (72 hours) in the presence of either HS-5 stroma or 3 primary AML MSCs. Apoptosis in response to PKC412 was measured with Annexin-V-FITC. ERG-induced cells were traced by DsRed (red bars); ERG-induced apoptotic cells were double positive (DsRed and Annexin V-FITC; white bars), whereas non-induced apoptotic cells were only positive for Annexin V-FITC (black bars). UT, untreated cells processed in parallel. The drug screen, CFSE, and stroma co-culture experiments were conducted in duplicates for both stably transfected K562 pTREERG CL9 and CL46 clones. Representative results for the CL9 clone are plotted in A) and B). The error bars denote the standard deviation.
ERG-induced and non-induced cells (Figure 3A and 3B). Addition of a CD44 blocking antibody completely abrogated the spindle shape formation in ERG-induced cells, whereas IgG alone did not elicit any morphological changes. ERG-induced cells were then treated with PKC412 to enhance spindle shape formation. In this case, antibody blocking of CD44 inhibited spindle shape formation and reduced cell adhesion by $75 \%$, whereas IgG antibody had no effect. Thus, ERG-induced spindle shape formation is mediated by CD44. We also measured apoptosis to determine the effect of CD44 block on ERGinduced cells. Only marginal changes in apoptosis (Figure 2A) were observed, affirming that CD44's main function is to aid cell-to-cell adhesion of in ERG-induced cells. Thus, disruption of ERG-mediated adhesion via a CD44 block may be of therapeutic significance in combination with current treatment protocols.

\section{Overexpression of ERG involves ERK 1/2 phosphorylation}

Current evidence supports the notion that, in cancer, MET (as opposed to EMT) is turned on in malignant cells to promote cell proliferation, migration, and evasion from apoptosis[18]. Genome-wide studies demonstrated that ERG activates the RAS/ERK pathway in a transgenic ERG leukemic model[11], and regulates cell migration in prostate cells[34]. Therefore, we sought to determine if ERG overexpression affected the phosphorylation levels of ERK1/2. Potent ERG overexpression induced higher pan ERK levels compared to non-induced cells, and the effect was enhanced with PKC412 and Ara-C treatment (Figure 4). Endogenous levels of phosphorylated ERK1/2 in ERG-induced cells were also elevated (including with Ara-C treatment), whereas no differences were observed in cells treated with PKC412. The lack of phosphorylation changes in ERK1/2 in cells treated with PKC412 suggests that other residues may be phosphorylated to enhance ERK signal transduction in ERG-induced cells. The upregulated levels of pan and phosphorylated ERK1/2 in ERG-induced cells indicate that signaling is upstream leading to adhesion and resistance to PKC412 and Ara-C treatment.

\section{Overexpression of ERG generates genomic instability}

Due to the severe downregulation of DNA repair gene expression in ERG overexpressing cells (Table 1) and in prostate cancer studies[14], we examined ERG overexpressing cells for the presence of DSBs by performing a neutral comet assay[24], with and without Ara-C or PKC412 treatments. The percentage of DNA in the comet tail reflects the number of DSBs. Noninduced cells displayed, on average, 30\% tail DNA, 
whereas ERG-induced cells harbored a significant greater amount (45-50\%) (Figure 5). Treatment of non-induced cells with Ara-C or PKC412 increased the percent tail DNA, as expected, to $50 \%$ compared to untreated controls. However, ERG-induced cells treated with the cytotoxic drugs showed a significant reduction of DSBs (10\% tail DNA with Ara-C and 30\% with PKC412). We

A.
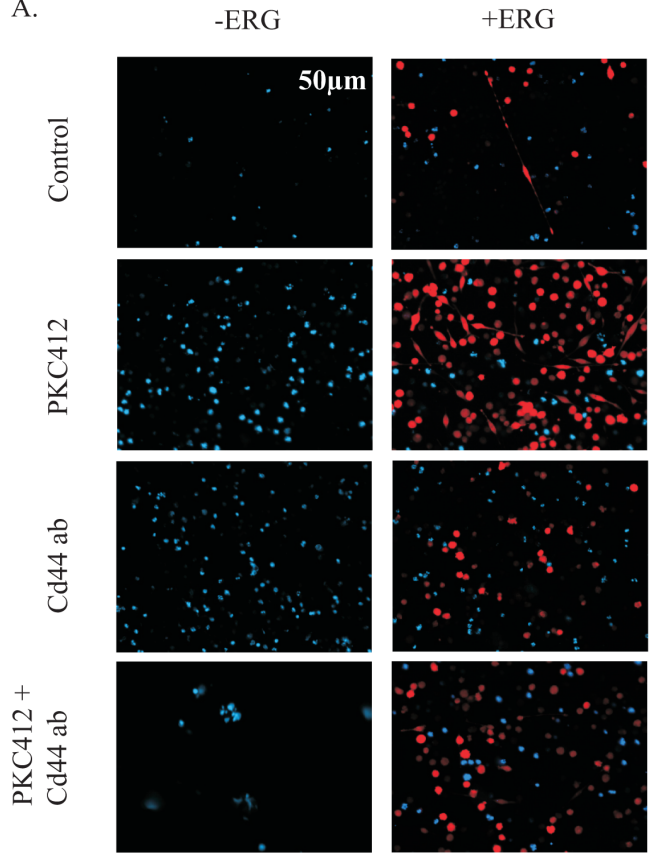

B.

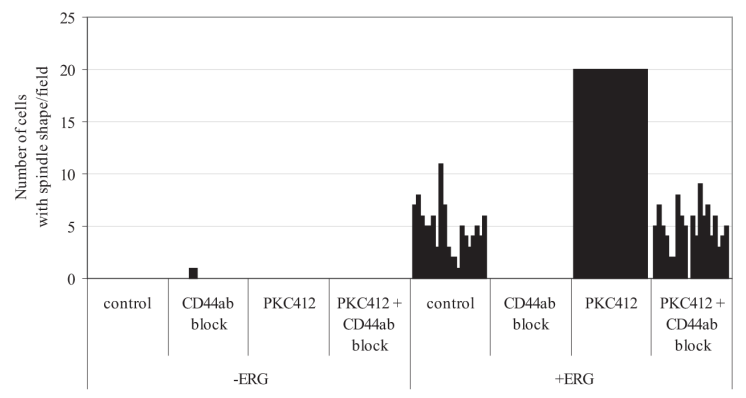

Figure 4: Spindle shape formation induced by ERG overexpression is inhibited by a CD44 blocking antibody. A) Representative fluorescence images data of K562 leukemia ERG-induced (+ERG) and non-induced (-ERG) cells after treatment for 72 hours with PKC412, blocking anti-CD44 (1:500 dilution) antibody alone, or the combined PKC412 and anti-CD44 (1:500 dilution) antibody. ERG-induced cells express DsRed and their nuclei were stained with DAPI. B) Graph of cells (counts) developing spindle shape formation for ERGinduced (+ERG) and non-induced cells (-ERG). ERG-induced and non-induced cells were treated with PKC412 to promote ERG dependent spindle-shape formation. The combination of ERG induction and PKC412 treatment induced potent spindle shaped cells in numbers greater than 20/field, which exceeds the error probability of an accurate cell count number. Thus, an upper limit of 20 spindle shaped cells/field was set. The graph represents the results of one of two experiments. attribute the reduction of \% tail DNA in ERG-induced cells treated with PKC412 to the activation of alternate DNA repair mechanisms, such as non-homologous endjoining, possibly to sustain high DNA replication rates (proliferative growth). Taken together, our data indicate that upon ERG induction, the oncogene generates increased DNA damage (DSBs) and that the effect may be reversed by treatment with Ara-C and PKC412. These observations complement the observed expansion of DsRed in ERG-induced cells and the enhanced proliferative rate in the presence of PKC412 (Figure 2A and $\mathrm{B}$ ). Hence, aberrant DNA repair may contribute to the multifactorial drug-mediated resistance in high ERG expressers in leukemia.

\section{DISCUSSION}

Herein we show that in leukemia cells ERG overexpression promotes a mesenchymal-like gene expression signature that includes: CD24, CD44, ITGA10, ITGB5, ITGB3, ITGA2B and the morphogenic-associated genes, such as SHANK3, TYROBP. These results are in line with the positive correlation of ERG-induced mesenchymal-like gene signature in leukemia with prostate carcinoma and chemotherapy-treated invasive breast tissues that validated our model system. We propose that, irrespective of cell type, high ERG expressing
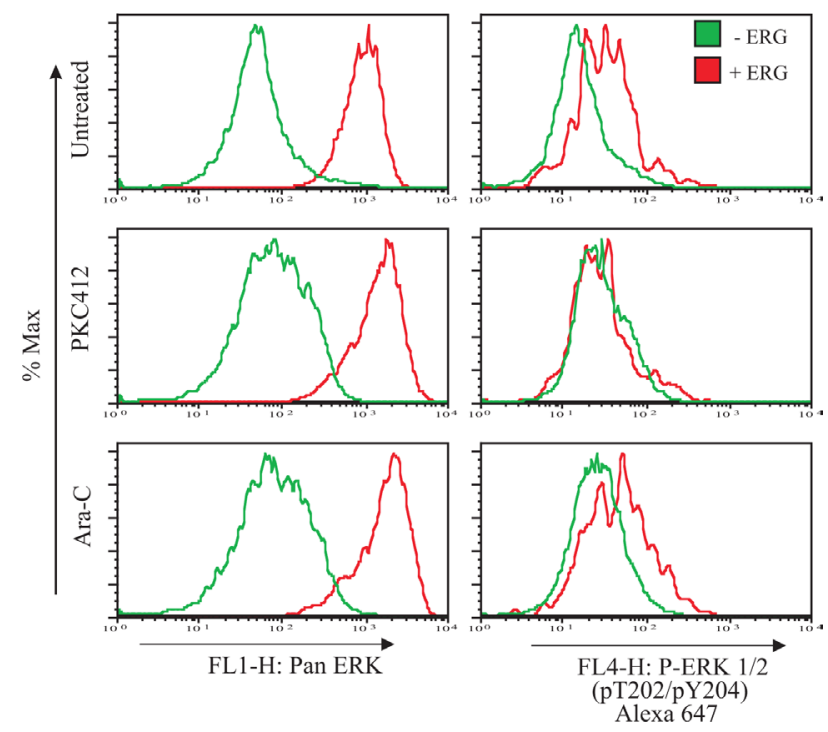

Figure 5: Intracellular staining of pan and phosphorylated ERK1/2 levels increase in cells with ERG overexpression. ERG-induced (+ERG) and noninduced (-ERG) cells were probed for intracellular levels of pan ERK1/2 and P-ERK1/2 (pT202/p204), left and right panels, respectively. To determine the extent of ERK activation, ERGinduced (+ERG) and non-induced cells (-ERG) were treated with either PKC412 or Ara-C for 24 hours. Plotted is a representative graph for intracellular probing for pan and phosphorylated levels of ERK1/2 and conducted in duplicate and duplicate in K562 pTRE-ERG clones, CL9 and CL46 clones. 
cells may initiate a malignant phenotype by promoting adhesion thereby enhancing the likelihood of escaping chemotherapeutic agents.

Through fluorescent tracking, we have also demonstrated that ERG overexpression in leukemia cells confers proliferative growth advantage at twice the rate of non-induced cells, whilst mediating the development of spindle shape morphology. In our stable phenotypic model of ERG overexpression, these proliferative features allowed for an even greater ERG-induced cell expansion in co-culture with the cell line HS-5 and in co-culture with primary bone marrow derived stroma from AML. Thus, enhanced adhesion of ERG overexpressing cells to the stromal bed may exploit direct interactions with stroma cells in order to increase cell survival. In the presence of cytotoxic drugs, the percentage of ERG-induced cells treated with PKC412 was enhanced, although the rates of
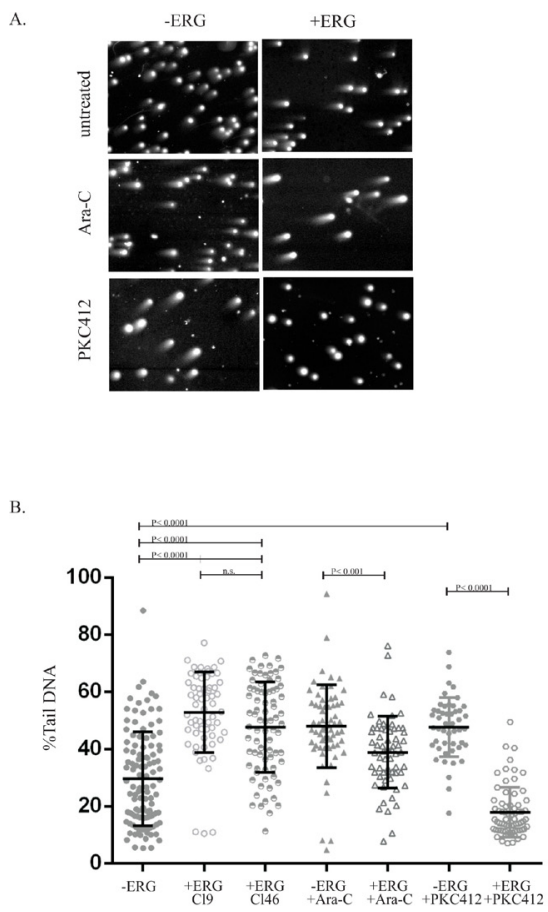

Figure 6: ERG-induced cells result in increased genomic instability and enhanced DNA repair in the presence of Ara-C and PKC412. ERG-induced (+ERG) and non-induced (-ERG) cells were treated with PKC412 or Ara-C for 96-hours and then analyzed by the neutral comet assay to detect the extent of DNA double strand breaks. A) Representative agarose smears of ERG-induced and non-induced cells stained with DAPI in the presence of Ara-C or PKC412. B) Quantification of \%Tail DNA was determined following treatment with Ara-C and PKC412 for 96-hours in ERG-induced (+ERG) and non-induced (-ERG) cells. The \%Tail DNA was determined for non-induced cells $(n=110)$, ERG-induced cells (CL9 and CL46, $n=63$ and $n=78$, respectively), non-induced cells treated with Ara-C $(n=63)$, ERG-induced cells treated with Ara-C $(n=62)$, non-induced cells treated with PKC412 ( $\mathrm{n}=48)$, and ERG-induced cells treated with PKC412 ( $\mathrm{n}=63)$. Statistical tests were performed using oneway ANOVA (Tukey test for multiple comparisons). proliferation were not altered (data not shown). In addition the drug resistance promoted directly by ERG, stroma may provide additional apoptotic protection from cytotoxic drugs.

ERG-induced leukemia cells treated with other PKC or WNT targeting drugs, such as LY294002, rapamycin and ICG-001, promoted expansion of ERG-induced cells rather than inducing apoptosis. This indicates that apoptotic evasion is another feature of ERG-induced survival, particularly under cytotoxic stress, possibly through the inhibition of PKC or WNT signaling pathways. Hence, it seems unlikely that targeted therapy through these drugs will benefit high ERG expressers. Conversely, nilotinib, PD325901, 5-azacytidine, dasatanib, were effective at depleting DsRed ERG positive cells. Of the agents screened, nilotinib induced apoptosis most effectively in ERG-induced cells.

Our treatment with a CD44 blocking antibody completely abrogated the spindle shape phenotype, with minimal apoptotic effects. Due to antigen specificity, blocking monoclonal antibodies have proven to be effective when combined with chemotherapy, such as rituximab for B-Cell lymphoma, trastuzumab for HER2positive cancer, cetuximab for colorectal carcinoma, and alemtuzumab for chronic lymphocytic leukemia. CD44, in particular, is believed to prevent homing of leukemia stem cells to the bone marrow, thus representing an attractive target for AML therapy[38]. This approach may also be applicable to the subgroup of high ERG expressers in AML in conjunction with other effective drug therapies, such as nilotinib.

The acquisition of drug resistance though activation of ERK $1 / 2$ is a common feature of oncogenes. ERK $1 / 2$ and downstream targets are regulated by ERG overexpression in the normal prostate cell line RWPE-1 through the ERG target PLAU[34]. Our results concur with these studies, since ERG overexpression resulted in greater amounts of phosphorylated ERK1/2 than in non-induced cells. ERGinduced cells treated with PKC412 led to an increase of pan ERK levels. Surprisingly, phosphorylated levels of pT202/Y204 were not detected, despite the remarkable expansion of ERG-induced cells and the enhanced spindle morphology. Thus, it is possible that either shunting to a different pathway branch occurred, or that phosphorylation took place at different ERK residues that were not recognized by the Y202/T204 antibody. That activation of the ERK1/2 pathway constitutes a critical step in the acquisition of drug resistance in ERG-induced cells is upstream, is further supported by our data with Ara-C, which revealed diminishing levels of ERK1/2 phosphorylation.

In prostate cancer, genomic mutations of DNA repair-associated genes, such as polymerase iota, were positively correlated with TMPRSS2-ERG positive tumors[39], which associates ERG overexpression with the DNA damage response. In ERG positive cells, the 
DNA repair genes PARP and DNA-PKs were also reported to co-immunoprecipitate with ERG. Likewise, inhibitors of PARP and DNA-PKs were found to impinge on ERG function, since the inhibitor olaparib (AZD2281) had no effect on ERG-mediated cellular proliferation but blocked ERG-mediated cell invasion[37]. In our current study, AZD2281 treatment did not affect apoptotic levels in ERG positive cells but blocked spindle shape formation. We also observed increased levels of DSB in ERGinduced leukemia cells, as previously reported in prostate cancer cell lines[14]. Also, in our leukemia model, ERG under-expressed genes included ATM, CHEK1 and ATR, which are key genes in the initiation of homologydependent DNA repair. Surprisingly, addition of PKC412 elicited a remarkable lessening in genomic instability in ERG-induced cells, suggesting either a restoration in DNA repair gene expression or a shift from homologous recombination to other types of DNA repair, including non homologous end joining (NHEJ). The latter hypothesis is supported by ERG-induced XRCC5, a key NHEJassociated gene and significantly ranked with high ERG levels in prostate mRNA expression datasets (Supplement Figure $1 \mathrm{~A}$ and $1 \mathrm{C}$ ). Hence, it is possible that a shift from homologous recombination to a NHEJ pathway of DNA repair may contribute to the escape from apoptosis and drug resistance. Further studies would be required to determine the precise repair processes coordinated by ERG in leukemia.

In conclusion, our K562 ERG-induced cell model system unraveled an ERG mesenchymal-like gene signature that overrides disease entities and reflects a general pattern of ERG-driven malignancies. We highlight, a number of changes in gene transcription in key pathways responsible for drug resistance, and provide novel targets for further validation and development in order to achieve custom tailored treatment strategies for the high ERG subgroup of leukemia.

\section{MATERIALS AND METHODS}

\section{Cells and drug treatment}

Cultures of K562 pTRE-ERG doxycycline (DOX) inducible clones were grown in 10\% FCS RPMI and seeded at $1 \times 105$ cells $/ \mathrm{ml}$, as previously described[21]. K562 pTRE-ERG cells were stimulated with DOX (1 $\mu \mathrm{g} / \mathrm{ml})$ for 24 hours. Following this step, drug was administered in the presence and absence of DOX in two K562 pTRE-ERG clones (CL9 and CL46) for an additional 96 hours. Drug (company, concentration ranges) used in this study are as follows: TKI258 (Novartis, 10 $\mu \mathrm{M}$ ), PKC412 (LC Laboratories, $10 \mu \mathrm{M}$ ), rapamycin (LC Laboratories, $10 \mu \mathrm{M}$ ), cytarabine (Cell Pharm, $20 \mu \mathrm{g} / \mathrm{ml}$ ), quercetin (Sigma, $10 \mu \mathrm{M}$ ), PD0325901
(Selleck Chemicals, $10 \mu \mathrm{M}$ ), daunorubicin (Pfizer, 10 $\mathrm{nM}$ ), nilotinib and dasatinib (Bug laboratory, Frankfurt Germany, 10 nM), olaparib (AZD22811, Selleck Chemicals, $10 \mu \mathrm{M}$ ), LY294002 (Cayman Chemical, 10 $\mu \mathrm{M})$, 3-deazaneplanocin A (Cayman Chemical, $10 \mu \mathrm{M})$, ICG001 (Selleck Chemical, $10 \mu \mathrm{M}$ ), XAV393 (Sigma, 10 $\mu \mathrm{M}$ ), and 5-azacytidine (Sigma, $1 \mu \mathrm{M}$ ). Dose curves were determined by Water Soluable Tetrazolium salt (WST) cell viability assays to determine near IC50 concentrations for each drug in K562 cells.

For co-culture experiments, the HS-5 stroma original cell line was purchased from ATCC and grown in $20 \%$ FCS RPMI to $80 \%$ confluence. Stromal layers were seeded one day prior to co-culture. ERG-inducible cells were stimulated for an additional 24 hours with DOX and combined with HS-5 stroma in 10\% FCS RPMI for an additional 24 hour period before the addition of drugs. ERG-inducible cells and stroma were then co-cultured for an additional 4 days with and without DOX to allow cell morphology changes to take place.

For the counting of spindle shaped cells, K562 pTRE-ERG CL9 and CL46 cells were seeded onto culture dishes with $2 \mathrm{~mm}$ grid plates and stimulated with DOX for six days. Two independent experiments with blind labels in duplicate were performed for each experimental group. Rabbit monoclonal anti-CD44 (H-CAM, Millipore) and anti-mouse IgG (Cell Signaling) were used in cell adhesion experiments at 1:500 dilution.

\section{Microarray expression and bioinformatic analyses}

Three independent stably transfected clones (CL4, CL9, CL46) of K562 pTRE-ERG cells were grown in the presence and absence of DOX (ERG-induced and non-induced) for long-term culture ( 8 days). Total RNA was extracted from ERG-induced and non-induced cells and hybridized using Affymetrix U133-plus chip. Data analysis was performed with Gene Spring 4.2 (Agilent Technologies). Differential expression of genes from biological triplicates (CL4, CL9, CL46) that displayed an over- and under-expression of 2-fold or greater between ERG-induced and non-induced cells were used for further analysis. Microarray data have been deposited in the Gene Expression Omnibus Database. To determine enriched biological terms, over- and under-expressed candidate gene lists were uploaded to the DAVID Bioinformatics server (http://david.abcc.ncifcrf.gov), which was employed to group and define enriched gene ontology terms[22].

\section{Comparative gene expression analyses}

Differential microarray dataset obtained from clones CL4, CL46, and CL9 (+/- DOX) were uploaded to the Oncomine Cancer Profiling Database (www.oncomine. 
org). Co-expression of ERG over-expressed and underexpressed genes were compared with leukemia, breast and prostate datasets which were obtained directly through the Oncomine 3.0 software.

\section{Quantitative RT PCR}

Quantitative RT PCR was conducted with Sybr green master mix (Fermentas) and analyses were conducted as previously described[21]. Relative mRNA expression was plotted as fold changes relative to uninduced cells from two independent pTRE-ERG CL9 and CL46 clones.

\section{Patient material}

To harvest mesenchymal stroma cells, the mononuclear fraction of bone marrow was cultured as previously described[23]. Informed consent of patient material was carried out according to the Declaration of Helsinki.

\section{Immunofluorescence and flow cytometry}

Live ERG-inducible adherent cells were stained with primary rabbit polyclonal anti-WNT11 (Novus Biologicals) and secondary anti-rabbit IgG (Alexa Fluor 488 conjugated) antibodies. Annexin V-FITC (BD Biosciences, San Jose, CA) staining was carried out according to manufacturer's instructions. Carboxyfluorescein diacetate succinimidyl ester, CFSE (Cayman Chemical) was used at 1:400 dilution and staining was conducted according to manufacturer's instructions.

To capture endogenous levels of pan ERK and ERK (pT202/pY204), ERG-induced cells were DOX stimulated for three days followed by 24-hour drug treatment (Ara-C or PKC412). Briefly, for intracellular staining, K562 pTRE-ERG cells $(5 \times 105$ cells $/ \mathrm{ml})$ were fixed with $150 \mu \mathrm{l}$ Phosflow fix buffer I (BD biosciences) at $37^{\circ} \mathrm{C}$, washed with PBS, resuspended in $200 \mu$ cold methanol for 10 min, blocked by PBS with $1 \%$ BSA, and labeled with the primary antibody (1:100 dilution) for $15 \mathrm{~min}$ at $4^{\circ} \mathrm{C}$. To detect endogenous ERK levels the $\alpha$-mouse pan ERK and phospho Alexa $\alpha$-mouse 647 (pT202/pY204) antibodies were used. When required, secondary antibody, antimouse IgG (Alexa Fluor 488 conjugated), was used for labeling. The three antibodies were purchased from Cell Signaling (Davers, MA). The labeled cells were measured by flow cytometry and analysed using Flowjo software. All flow cytometry techniques were conducted on a Becton Dickinson FACSCalibur instrument.

\section{Comet assay}

Double strand DNA breakage was measured through the neutral comet assay[24]. ERG-induced cells were stimulated with DOX for 24 hours prior to the addition of Ara-C or PKC412. Following a 72-hour drug treatment, $1 \times 105$ cells were suspended into melted $1 \%$ agarose (1:10 dilution) and smeared onto a precoated 1\% agarose glass slide. Slides were then submerged into lysis buffer (2.5 M NaCl, 0.1 M EDTA, $10 \mathrm{mM}$ Tris pH7.5, $1 \%$ Triton X-100, 1\% sodium lauryl sarcosine, $0.5 \mathrm{mg} /$ $\mathrm{ml}$ proteinase $\mathrm{K}$ ) overnight at $4^{\circ} \mathrm{C}$. Slides were rinsed in $\mathrm{TE}$ buffer, equilibrated in an electrophoresis chamber with neutral electrophoresis buffer for 30 minutes and then electrophoresis was conducted for 25 minutes at 25 volts. Slides were then submerged in distilled water, stained with 4',6-diamidine-2-phenylindol dihydrochloride (DAPI, 1.4 $\mu \mathrm{M})$, fixed in $70 \%$ ethanol and air dried. Experiments were conducted in duplicate, in both CL9 and CL46 clones, with four to five 100x magnification images for each treatment. The fluorescence intensities of the comet head and comet tail of all cells (63 to 110) in each image were scored. Percent DNA in tail and head: the total fluorescence intensity within the delineated comet tail was divided by the total comet intensity (comet head plus comet tail intensity) and multiplied by 100 . Measurements were conducted with the Image J software and Comet Tail analysis plugin (rsbweb.nih.gov/IJ).

\section{ACKNOWLEDGEMENTS:}

We thank Dr. Albino Bacolla for his expertise on DNA repair and for the critical review of this manuscript. This work was supported by a grant from Deutsche Krebshilfe to Claudia D. Baldus.

\section{REFERENCES}

1. Loughran SJ, Kruse EA, Hacking DF, De Graaf CA, Hyland CD,Willson TA, Henley KJ, Ellis S, Voss AK, Metcalf D, Hilton DJ, Alexander WS, Kile BT. The transcription factor Erg is essential for definitive hematopoiesis and the function of adult hematopoietic stem cells. Nature Immunology. 2008; 9: 810-819.

2. Salek-Ardakani S, Smooha G, De Boer J, Sebire NJ, Morrow M, Rainis L, Lee S, Williams O, Izraeli S, Brady HJM. ERG is a megakaryocytic oncogene. Cancer Research. 2009; 69: 4665-4673.

3. Ichikawa H, Shimizu K, Hayashi Y, Ohki M. An RNAbinding protein gene, TLS/FUS, is fused to ERG in human myeloid leukemia with $\mathrm{t}(16 ; 21)$ chromosomal translocation. Cancer Research. 1994; 54: 2865-2868.

4. Sorensen PHB, Lessnic SL, Lopez-Terrada D, Liu XF, Triche TJ, Denny CT. A second Ewing's sarcoma translocation, $\mathrm{t}(21 ; 22)$, fuses the EWS gene to another ETS- 
family transcription factor, ERG. Nature Genetics. 1994; 6: 146-151.

5. Tomlins SA, Rhodes DR, Perner S, Dhanasekaran SM, Mehra R, Sun X-W, Varambally S, Cao X, Tchinda J, Kuefer R, Lee C, Montie JE, Shah RB, Pienta KJ, Rubin MA, Chinnaiyan AM. Recurrent fusion of TMPRSS2 and ETS transcription factor genes in prostate cancer. Science. 2005; 310: 644-648.

6. Perner S, Demichelis F, Beroukhim R, Schmidt FH, Mosquera J-M, Setlur S, Tchinda J, Tomlins SA, Hofer MD, Pienta KG, Kuefer R, Vessella R, Sun X-W, Meyerson M, Lee C, Sellers WR, Chinnaiyan AM, Rubin MA. TMPRSS2:ERG fusion-associated deletions provide insight into the heterogeneity of prostate cancer. Cancer Research. 2006; 66: 8337-8341.

7. Marcucci G, Baldus CD, Ruppert AS, Radmacher MD, Mro K, Whitman SP, Kolitz JE, Edwards CG, Vardiman JW, Powell BL, Baer MR, Moore JO, Perrotti D, Caligiuri MA, Carroll AJ, Larson RA, De Chapelle A, Bloomfield CD. Overexpression of the ETS-Related Gene, ERG, predicts a worse outcome in acute myeloid leukemia with normal karyotype: A Cancer and Leukemia Group B Study. Journal of Clinical Oncology. 2005; 23: 9234-9242.

8. Baldus CD, Burmeister T, Martus P, Schwartz S, Gökbuget N, Bloomfield CD, Hoelzer D, Thiel E, Hofmann WK. High expression of the ETS transcription factor ERG predicts adverse outcome in acute T-lymphoblastic leukemia in adults. Journal of Clinical Oncology. 2006; 24: 4714-4720.

9. Tsuzuki S, Taguchi O, Seto M. Promotion and maintenance of leukemia by ERG. Blood. 2011; 117: 3858-3868.

10. Thoms JAI, Birger Y, Foster S, Knezevic K, Kirschenbaum Y, Jonquieres G, Spensberger D, Wong JW, Oram SH, Kinston J, Groner Y, Lock R, Mackenzie KL, Göttgens B, Izraeli S, Pimanda JE. ERG promotes T-acute lymphoblastic leukemia and is transcriptionally regulated in leukemic cells by a stem cell enhancer ERG promotes T-acute lymphoblastic leukemia and is transcriptionally regulated in leukemic cells by a stem cell enhancer. Blood. 2011; 117: 7079-7089.

11. Goldberg L, Tijssen MR, Birger Y, Hannah RL, Kinston SJ, Schutte J, Beck D, Knezevic K, Schiby G, Jacob-Hirsch J, Biran A, Kloog Y, Marcucci G, Bloomfield CD, Aplan PD, Pimanda JE, Gottgens B, Izraeli S. Genome-scale expression and transcription factor binding profiles reveal therapeutic targets in transgenic ERG myeloid leukemia. Blood. 2013: E-print.

12. Tomlins SA, Laxman B, Varambally S, Cao X, Yu J, Helgeson BE, Cao Q, Prensner JR, Rubin MA, Shah RB, Mehra R, Chinnaiyan AM. Role of the TMPRSS2-ERG gene fusion in prostate cancer. Neoplasia. 2008; 10: 177188.

13. Gupta S, Iljin K, Sara H, Mpindi JP, Mirtti T, Vainio P, Rantala J, Alanen K, Nees M, Kallioniemi O. FZD4 as a mediator of ERG oncogene-induced WNT signaling and epithelial-to-mesenchymal transition in human prostate cancer cells. Cancer Research. 2010; 70: 6735-6745.

14. Brenner JC, Ateeq B, Li Y, Yocum AK, Cao Q, Asangani IA, Patel S, Wang X, Liang H, Yu J, Palanisamy N, Siddiqui J, Yan W, Cao X, Mehra, Sabolch A, Basrur V, Lonigro RJ, Yang J, Tomlins SA, Maher CA, Elenitoba-Johnson KSJ, Hussain M, Navone NM, Pienta KJ, Varambally S, Feng FY, Chinnaiyan AM. Mechanistic rationale for inhibition of poly(ADP-ribose) polymerase in ETS gene fusion-positive prostate cancer. Cancer Cell. 2011; 19: 664-678.

15. Haffner MC, De Marzo AM, Meeker AK, Nelson WG, Yegnasubramanian S. Transcription-induced DNA double strand breaks: both oncogenic force and potential therapeutic target? Clinical Cancer Research. 2011; 17: 3858-3864.

16. Sreenath TL, Dobi A, Petrovics G, Srivastava S. Oncogenic activation of ERG: A predominant mechanism in prostate cancer. Journal of Carcinogenesis. 2012; 11: 10-21.

17. Kalluri R, Weinberg RA. The basics of epithelialmesenchymal transition. Journal of Clinical Investigation. 2009; 119: 1420-1428.

18. Singh A, Settleman J. EMT, cancer stem cells and drug resistance: an emerging axis of evil in the war on cancer. Oncogene. 2010; 29: 4741-4751.

19. Hsu T, Trojanowska M, Watson DK. Ets proteins in biological control and cancer. J Cell Biochem. 2004; 91: 896-903.

20. Puissant A, Dufies M, Fenouille N, Ben Sahra I, Jacquel A, Robert G, Cluzeau T, Deckert M, Tichet M, Chéli Y, Cassuto JP, Raynaud S, Legros L, Pasquet JM, Mahon FX, Luciano F, Auberger P. Imatinib triggers mesenchymallike conversion of CML cells associated with increased aggressiveness. Journal of Molecular Cell Biology. 2012; 4: 207-220.

21. Mochmann LH, Bock J, Ortiz-Tanchez J, Schlee C, Bohne A, Hofmann W-K, Thiel E, Baldus CD. Genome-wide screen reveals WNT11, a non-canonical WNT gene, as a direct target of ETS transcription factor ERG. Oncogene. 2011; 30: 2044-2056.

22. Jiao X, Sherman BT, Huang DW, Stephens R, Baseler RW, Lane HC, Lempicki RA. DAVID-WS: a stateful web service to facilitate gene/protein list analysis. Bioinformatics. 2012; 28: 1805-1806.

23. Blau O, Hofmann W-K, Baldus CD, Thiel G, Serbent V, Schümann E, Thiel E, Blau IW. Chromosomal aberrations in bone marrow mesenchymal stroma cells from patients with myelodysplastic syndrome and acute myeloblastic leukemia. Experimental Hematology. 2007; 35: 221-229.

24. Olive PL, Banáth JP. The comet assay: a method to measure DNA damage in individual cells. Nature Protocols. 2006; 1 : 23-29.

25. Oliveras-Ferraros B, Corominas-Faja C, Cufí S, VazquezMartin A, Martin-Castillo B, Iglesias JM, Lopez-Bonet E, Martin ÁG, and Menendez JA. Epithelial-to-mesenchymal transition (EMT) confers primary resistance to trastuzumab. 
Cell Cycle. 2012; 11: 4020-4032.

26. Bock J, Mochmann LH, Schlee C, Farhadi-Sartangi N, Göllner S, Müller-Tidow C, Baldus CD. ERG transcriptional networks in primary acute leukemia cells implicate a role for ERG in deregulated kinase signaling. PloS ONE. 2013; 8: e52872.

27. Wang Z, Li SS. A new player in EMT. Cell Adhesion and Migration. 2010; 4: 176-179.

28. Cao Q, Yu J, Dhanasekaran SM, Kim JH, Mani R-S, Tomlins SA, Mehra R, Laxman B, Cao X, Kleer CG, Varambally S, Chinnaiyan A. Repression of E-cadherin by the polycomb group protein EZH2 in cancer. Oncogene. 2008; 27: 7274-7284.

29. Grasso CS, Wu Y-M, Robinson DR, Cao X, Dhanasekaran SM, Khan AP, Quist MJ, Jing X, Lonigro RJ, Brenner JC, Asangani IA, Ateeq B, Chun SY, Siddiqui J, Sam L, Anstett M, Mehra R, Prensner JR, Palanisamy N, Ryslik GA, Vandin F, Raphael BJ, Kunju LP, Rhodes DR, Pienta KJ, Chinnaiyan AM, Tomlins SA. The mutational landscape of lethal castration-resistant prostate cancer. Nature. 2012; 487: 239-243.

30. Stickeler E, Pils D, Klar M, Orlowsk-Volk M, Zur Hausen A, Jäger M, Watermann D, Gitsch G, Zeillinger R, Tempfer CB. Basal-like molecular subtype and HER4 up-regulation and response to neoadjuvant chemotherapy in breast cancer. Oncology Reports. 2011; 26: 1037-1045.

31. Rickman DS, Soong TD, Moss B, Mosquera JM, Dlabal J, Terry S, MacDonald TY, Tripodi J, Bunting K, Najfeld V, Demichelis F, Melnick AM, Elemento O, and Rubin MA. Oncogene-mediated alterations in chromatin conformation. PNAS. 2012; 109: 9083-9088.

32. Gupta S, Iljin K, Sara H, Mpindi JP, Mirtti T, Vainio P. FZD4 as a Mediator of ERG Oncogene - Induced WNT Signaling and Epithelial-to-Mesenchymal Transition in Human Prostate Cancer Cells. Cancer Research. 2010; 70: 6735-6745.

33. Carver BS, Tran J, Gopalan A, Chen Z, Shaikh S, Carracedo A, Alimonti A, Nardella C, Varmeh S, Scardino PT, Cordon-Cardo C, Gerald W, Pandolfi PP. Aberrant ERG expression cooperates with loss of PTEN to promote cancer progression in the prostate. Nature Genetics. 2009; 41: 619624.

34. Hollenhorst PC, Ferris MW, Hull MA, Chae H, Kim S, Graves BJ. Oncogenic ETS proteins mimic activated RAS/ MAPK signaling in prostate cells. Genes \& Development. 2011; 25: 2147-2157.

35. Patel AG, Sarkaria JN, Kaufmann SH. Nonhomologous end joining drives poly (ADP-ribose) polymerase (PARP) inhibitor lethality in homologous. PNAS. 2011; 108: 3406 3411.

36. Yu J, Yu J, Mani R-S, Cao Q, Brenner CJ, Cao X, Wang $\mathrm{X}$, Wu L, Li J, Hu M, Gong Y, Cheng H, Laxman B, Vellaichamy A, Shankar S, Li Y, Dhanasekaran SM, Morey R, Barrette T, Lonigro RJ, Tomlins SA, Varambally S, Qin
ZS, Chinnaiyan AM. An integrated Network of Androgen Receptor, Polycomb, and TMPRSS2-ERG Gene Fusions in Prostate Cancer Progression. Cancer Cell. 2010; 17: 443454.

37. Brenner JC, Ateeq B, Li Y, Yocum AK, Cao Q, Asangani IA, Patel S, Wang X, Liang H, Yu J, Sabolch N, Siddiqui J, Yan W, Cao X, Mehra R, Sabolch A, Basrur V, Lonigro RJ, Yang J, Tomlins SA, Maher CA, Elenitoba-Johnson KSJ, Hussain M, Navone NM, Pienta KJ, Varambally S, Feng FY, Chinnaiyan AM. Mechanistic Rationale for Inhibition of Poly(ADP-Ribose) Polymerase in ETS Gene Fusion-Positive Prostate Cancer. Cancer Cell. 2011; 19: 664-678.

38. Majeti R. Monoclonal antibody therapy directed against human acute myeloid leukemia stem cells. Oncogene. 2011; 30: 1009-1019.

39. Luedeke M, Linnert CM, Hofer MD, Surowy HM, Rinckleb AE, Hoegel J, Kuefer R, Rubin MA, Vogel W, Maier C. Predisposition for TMPRSS2-ERG fusion in prostate cancer by variants in DNA repair genes. Cancer Epidemiology, Biomarkers \& Prevention. 2009; 18: 3030-3035. 